\title{
Polynomial energy decay of a wave-Schrödinger transmission system
}

\section{Chengqiang Wang ${ }^{*}$ (D)}

\section{"Correspondence:}

cqwung@foxmail.com

School of Mathematics, Chengdu

Normal University, Chengdu, China

\begin{abstract}
We study in this paper a wave-Schrödinger transmission system for its stability. By analyzing carefully Green's functions for the infinitesimal generator of the semigroup associated with the system under consideration, we obtain a useful resolvent estimate on this generator which can be applied to derive the decaying property. Our study is inspired by L. Lu \& J.-M. Wang [Appl. Math. Lett., 54:7-14, 2016] whose energy decay result is improved upon in our paper. Our method, different from the one used in the previous reference, can be adapted to study stability problems for other 1-D transmission systems.
\end{abstract}

MSC: Primary 35Q41; secondary 35B30; 35B35; 35C20; 35L10; 35P05

Keywords: Wave-Schrödinger transmission system; Polynomial energy decay; Resolvent estimate; Green's functions

\section{Introduction}

Thanks to its wide applicability, the Schrödinger equation

$$
\mathrm{i} \partial_{t} u+\Delta u+f(\nabla u, u, x, t)=0, \quad(x, t) \in \mathbb{R}^{n} \times \mathbb{R},
$$

where $\Delta=\sum_{j=1}^{n} \frac{\partial^{2}}{\partial x_{j}^{2}}$ is the Laplacian on $\mathbb{R}^{n}$, has been receiving extensive attention from the mathematical control community; see [2-8] and the references cited therein. Specifically, the systems described by the Schrödinger equation have received extensive studies for their stability in the past three decades. Among the vast references in this direction, Lagnese [9] proved a stability result via "connecting" it to the stability property of the plate equation $\partial_{t}^{2} u+\Delta^{2} u+$ l.o.t $=0$ (while the study of the stability and stabilization of the plate equation has a relatively long history). Machtyngier and Zuazua [4] studied the boundary and internal stabilization problem via the multiplier method (the main idea has originated from stability studies for wave equations). In [7, 10], some collocated boundary stabilization problems were investigated. Zuazua [2] provided a nice survey on the recent studies on the control properties for the Schrödinger equation.

This paper is devoted to the study of the stabilization of the Schrödinger equation via a damped wave equation through a common end point. More precisely, we are concerned

(c) The Author(s) 2018. This article is distributed under the terms of the Creative Commons Attribution 4.0 International License (http://creativecommons.org/licenses/by/4.0/), which permits unrestricted use, distribution, and reproduction in any medium, provided you give appropriate credit to the original author(s) and the source, provide a link to the Creative Commons license, and indicate if changes were made. 
in this paper with the system

$$
\begin{cases}\mathrm{i} \partial_{t} u+\partial_{x}^{2} u=0 & \text { in }(0,1) \times(0, \infty) \\ \partial_{t}^{2} v-\partial_{x}^{2} v+b \partial_{t} v=0 & \text { in }(0,1) \times(0, \infty) \\ u(1, \cdot)=v(1, \cdot)=u(0, \cdot)-k \partial_{t} v(0, \cdot) & \\ \quad=\partial_{x} v(0, \cdot)-\mathrm{i} k \partial_{x} u(0, \cdot)=0 & \text { in }(0, \infty)\end{cases}
$$

where $\mathrm{i}=\sqrt{-1}$ is the imaginary unit, and $k \in \mathbb{R} \backslash\{0\}$ and $b \in(0, \infty)$ are fixed arbitrarily. System (1.1) was recently studied by Lu and Wang [1] with the intension to understand better the transmission of dissipation effect from a damped wave equation to a dampingfree Schrödinger equation where the energy can be exchanged by $(1.1)_{1}$.

The natural phase space for system (1.1) is

$$
H=\left\{(f, g, h) \in L^{2}(0,1 ; \mathbb{C}) \times H^{1}(0,1 ; \mathbb{C}) \times L^{2}(0,1 ; \mathbb{C}) ; g(1)=0\right\} .
$$

Let us define an unbounded linear operator $A$ in $H$ by

$$
\left\{\begin{array}{c}
\mathcal{D}(A)=\left\{(f, g, h) \in H^{2}\left(0,1 ; \mathbb{C}^{2}\right) \times H^{1}(0,1 ; \mathbb{C})\right. \\
\left.f(1)=g(1)=h(1)=f(0)-k h(0)=g^{\prime}(0)-\mathrm{i} k f^{\prime}(0)=0\right\}, \\
A(f, g, h)=\left(\mathrm{i} f^{\prime \prime}, h, g^{\prime \prime}-b h\right), \quad \forall(f, g, h) \in \mathcal{D}(A) .
\end{array}\right.
$$

We can prove as in [1] that $A$ is the infinitesimal generator of a strongly continuous semigroup $\left\{e^{t A}\right\}$ on $H$. Therefore, (1.1) admits for every triple $\left(u^{0}, u^{1}, v^{0}\right) \in H$ a unique solution $(u, v) \in \mathbb{S}^{0}$; if further $\left(u^{0}, u^{1}, v^{0}\right) \in \mathcal{D}(A)$, then $(u, v) \in \mathbb{S}^{1}$. Here $\mathbb{S}^{0}$ and $\mathbb{S}^{1}$ are defined by

$$
\begin{aligned}
\mathbb{S}^{0}:= & \mathcal{C}\left([0, \infty) ; L^{2}(0,1)\right) \times\left[\mathcal{C}\left([0, \infty) ; H^{1}(0,1)\right) \cap \mathcal{C}^{1}\left([0, \infty) ; L^{2}(0,1)\right)\right] \text { and } \\
\mathbb{S}^{1}:= & {\left[\mathcal{C}\left([0, \infty) ; H^{2}(0,1)\right) \cap \mathcal{C}^{1}\left([0, \infty) ; L^{2}(0,1)\right)\right] } \\
& \times\left[\mathcal{C}\left([0, \infty) ; H^{2}(0,1)\right) \cap \mathcal{C}^{1}\left([0, \infty) ; H^{1}(0,1)\right)\right] .
\end{aligned}
$$

We associate with system (1.1) the following energy functional:

$$
E(t)=\frac{1}{2} \int_{0}^{1}\left(|u(x, t)|^{2}+\left|\partial_{x} v(x, t)\right|^{2}+\left|\partial_{t} v(x, t)\right|^{2}\right) d x, \quad \forall t \in[0, \infty)
$$

As indicated before, the study of this paper is directly inspired by [1]. And therefore, it is worth recalling the main results in [1] as follows.

Theorem A (see [1]) Let $A$ be defined as in (1.3), $E$ as in (1.6), and $H$ as in (1.2).

- $A$ is the infinitesimal generator of a strongly continuous semigroup $\left\{e^{t A}\right\}_{t \in[0, \infty)}$ of contractions on $H$. In particular, we have: For every triple $\left(u^{0}, v^{0}, v^{1}\right) \in H$, the boundary value problem (1.1) admits a unique solution $(u, v) \in \mathbb{S}^{0}$ such that $u(\cdot, 0)=u^{0}$, $v(\cdot, 0)=v^{0}$ and $\partial_{t} v(\cdot, 0)=v^{1} ;$ if, in addition, $\left(u^{0}, v^{0}, v^{1}\right) \in \mathcal{D}(A)($ see $(1.3))$, then $(u, v) \in \mathbb{S}^{1}$. 
- The spectrum $\sigma(A)$ of $A$ consists merely of eigenvalues of $A$, and is distributed as follows:

$$
\left.\begin{array}{l}
\lambda_{1 j}=-\frac{b}{2} \pm \frac{\sqrt{b^{2}-4 j^{2} \pi^{2}}}{2}+\mathcal{O}\left(j^{-1}\right), \\
\lambda_{2 j}=-\left|j-\frac{1}{2}\right|^{2} \pi^{2} \mathbf{i}+\mathcal{O}\left(j^{-1}\right), \quad \Re \mathfrak{e} \lambda_{2 j}<0
\end{array}\right\}, \quad \text { as } j \nearrow \infty .
$$

- $E(t) \searrow 0$ as $t \nearrow \infty$.

Note especially that Lu and Wang [1] proved that $E(t)$ decreases to 0 as $t \rightarrow+\infty$. But due to the fact that $\lim _{j \rightarrow \infty} \Re \mathfrak{R} \lambda_{2 j}=0, E(t)$ cannot decay uniformly (see the last section of the paper for a brief proof of this statement). Recently, the non-uniform decay properties have been investigated extensively in the literature for PDEs; see [11, 12]. Our main result gives a more accurate decay rate for the energy $E(t)$.

Theorem 1.1 Let E, defined as in (1.6), be the energy associated with system (1.1). There exists $M \in(0, \infty)$ such that, for every solution $(u, v) \in \mathbb{S}^{1}$ with $u(\cdot, 0)=u^{0}, v(\cdot, 0)=v^{0}$, and $\partial_{t} v(\cdot, 0)=v^{1}$,

$$
E(t) \leq \frac{M}{t+1}\left(\left\|u^{0}\right\|_{H^{2}(0,1)}^{2}+\left\|v^{0}\right\|_{H^{2}(0,1)}^{2}+\left\|v^{1}\right\|_{H^{1}(0,1)}^{2}\right), \quad \forall t \in[0, \infty) .
$$

By [13, Theorem 2.4], this theorem follows immediately from the following theorem.

Theorem 1.2 Let $A$ be defined by (1.3). There exists $C \in(0, \infty)$ such that ${ }^{\mathrm{a}}$

$$
\|R(\mathrm{i} \gamma ; A)\|_{\mathcal{L}(H)} \leq C\langle\lambda\rangle^{2}, \quad \forall \gamma \in \mathbb{R} .
$$

Throughout this paper, $C$ is a generic constant which can assume a different value at each occurrence.

The rest of the paper is organized as follows. With the aid of the idea of Green's functions, we provide in Sect. 2 an explicit formulae for the resolvent $R(\mathrm{i} \gamma ; A)$. The main results of this paper are proved in Sect. 3. Some concluding remarks are included in Sect. 4.

\section{Green's functions and the resolvent $R(\mathrm{i} \gamma ; A)$}

We would like to calculate in this section the resolvent $R(\mathrm{i} \gamma ; A)$ with $\gamma \in \mathbb{R}$ by using the idea of Green's functions. Let $(\phi, \psi, \eta) \in H$. Consider the equation $\left(\lambda \operatorname{id}_{H}-A\right)(f, g, h)=$ $(\phi, \psi, \eta)$ with $\operatorname{id}_{H}$ denoting the identity operator on $H$, or equivalently, the boundary value problem (BVP)

$$
\left\{\begin{array}{l}
f^{\prime \prime}+\lambda \mathrm{i} f=\mathrm{i} \phi, \quad h=\lambda g-\psi, \quad g^{\prime \prime}-\lambda(\lambda+b) g=-(\lambda+b) \psi-\eta \\
f(1)=g(1)=f(0)-k h(0)=g^{\prime}(0)-\mathrm{i} k f^{\prime}(0)=0 .
\end{array}\right.
$$


Denote by $F^{j}$ and $G^{j}, j=1,2,3$, the Green's functions for BVP (2.1). By using the idea of Green's functions, every solution $(f, g, h)$ to BVP $(2.1)$ can be expressed by

$$
\left.\begin{array}{rl}
f(x)= & \mathrm{i} \int_{0}^{1} F^{1}(x, \xi) \phi(\xi) d \xi+\int_{0}^{1} F^{2}(x, \xi) \psi(\xi) d \xi \\
& +\int_{0}^{1} F^{3}(x, \xi) \eta(\xi) d \xi \\
g(x)= & \int_{0}^{1} G^{1}(x, \xi) \phi(\xi) d \xi-(\lambda+b) \int_{0}^{1} G^{2}(x, \xi) \psi(\xi) d \xi \\
& -\int_{0}^{1} G^{3}(x, \xi) \eta(\xi) d \xi, \\
h(x)= & \lambda \int_{0}^{1} G^{1}(x, \xi) \phi(\xi) d \xi-\lambda(\lambda+b) \int_{0}^{1} G^{2}(x, \xi) \psi(\xi) d \xi \\
& -\lambda \int_{0}^{1} G^{3}(x, \xi) \eta(\xi) d \xi-\psi(x)
\end{array}\right\}, \quad \forall x \in[0,1] .
$$

The Green's functions for BVP (2.1) should assume the form

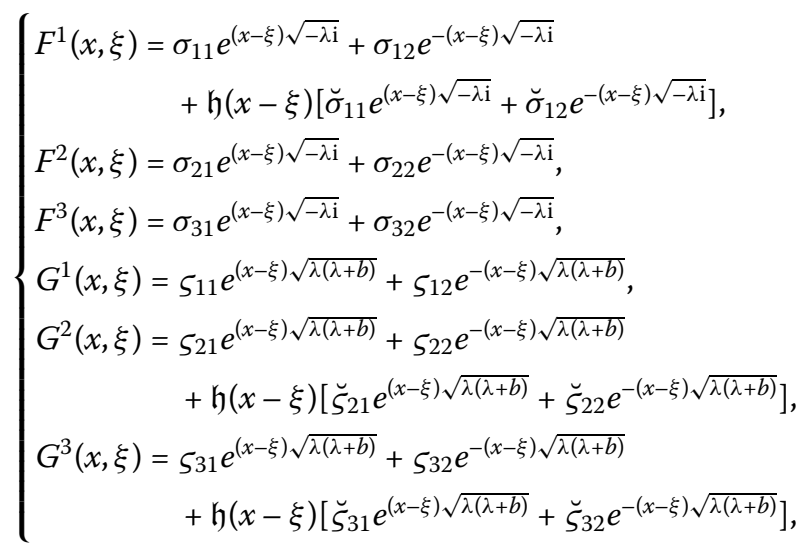

where $\mathfrak{h}$ is the Heaviside function, namely

$$
\mathfrak{h}(\mu)= \begin{cases}0 & \text { if } \mu \leq 0 \\ 1 & \text { if } \mu>0\end{cases}
$$

and the coefficients $\sigma_{j k}, \varsigma_{j k}(j=1,2,3, k=1,2), \breve{\sigma}_{11}, \breve{\sigma}_{12}, \breve{\zeta}_{j k}(j=2,3, k=1,2)$ are yet to be determined later (see (2.4), (2.5), (2.6), and (2.7)). The Green's functions should also satisfy

$$
\begin{aligned}
& f(1)=0 \quad \Longrightarrow \quad F^{1}(1, \xi)=F^{2}(1, \xi)=F^{3}(1, \xi)=0, \\
& g(1)=0 \quad \Longrightarrow \quad G^{1}(1, \xi)=G^{2}(1, \xi)=G^{3}(1, \xi)=0 \text {, } \\
& f(0)=k h(0) \quad \Longrightarrow \quad \mathrm{i} F^{1}(0, \xi)-\lambda k G^{1}(0, \xi) \\
& \left.\begin{array}{l}
=F^{2}(0, \xi)+k \lambda(\lambda+b) G^{2}(0, \xi)+k \delta_{0}(\xi) \\
=F^{3}(0, \xi)+\lambda k G^{3}(0, \xi)=0,
\end{array}\right\}, \quad \forall \xi \in[0,1] . \\
& g^{\prime}(0)=\mathrm{i} k f^{\prime}(0) \quad \Longrightarrow \quad k \partial_{x} F^{1}(0, \xi)+\partial_{x} G^{1}(0, \xi) \\
& =k \partial_{x} F^{2}(0, \xi)-\mathrm{i}(\lambda+b) \partial_{x} G^{2}(0, \xi) \\
& =k \partial_{x} F^{3}(0, \xi)-\mathrm{i} \partial_{x} G^{3}(0, \xi)=0
\end{aligned}
$$

This, together with the notion of Green's functions, implies

$$
\left\{\begin{aligned}
\breve{\zeta}_{21}+\breve{\zeta}_{22}=\breve{\zeta}_{31}+\breve{\zeta}_{32}=\breve{\sigma}_{11}+\breve{\sigma}_{12} & =0, \\
\breve{\zeta}_{21} \sqrt{\lambda(\lambda+b)}-\breve{\zeta}_{22} \sqrt{\lambda(\lambda+b)} & =\breve{\zeta} 31 \sqrt{\lambda(\lambda+b)}-\breve{\zeta}_{32} \sqrt{\lambda(\lambda+b)} \\
& =\breve{\sigma}_{11} \sqrt{-\lambda \mathrm{i}}-\breve{\sigma}_{12} \sqrt{-\lambda \mathrm{i}}=\frac{1}{2},
\end{aligned}\right.
$$




$$
\begin{aligned}
& \sigma_{11} e^{(1-\xi) \sqrt{-\lambda \mathrm{i}}}+\sigma_{12} e^{-(1-\xi) \sqrt{-\lambda \mathrm{i}}}=-\breve{\sigma}_{11} e^{(1-\xi) \sqrt{-\lambda \mathrm{i}}}-\breve{\sigma}_{12} e^{-(1-\xi) \sqrt{-\lambda \mathrm{i}}} \\
& =-\frac{1}{2 \sqrt{-\lambda \mathrm{i}}} \sinh ((1-\xi) \sqrt{-\lambda \mathrm{i}}), \\
& \left.\begin{array}{l}
\varsigma_{11} e^{(1-\xi) \sqrt{\lambda(\lambda+b)}}+\varsigma_{12} e^{-(1-\xi) \sqrt{\lambda(\lambda+b)}}=0, \\
\sigma_{11} e^{-\xi \sqrt{-\lambda \mathrm{i}}}+\sigma_{12} e^{\xi \sqrt{-\lambda \mathrm{i}}}+\varsigma_{11} \mathrm{i} \lambda k e^{-\xi \sqrt{\lambda(\lambda+b)}}+\varsigma_{12} \mathrm{i} \lambda k e^{\xi \sqrt{\lambda(\lambda+b)}}=0,
\end{array}\right\}, \quad \forall \xi \in[0,1], \\
& \sigma_{11} k \sqrt{-\lambda \mathrm{i}} e^{-\xi \sqrt{-\lambda \mathrm{i}}}-\sigma_{12} k \sqrt{-\lambda \mathrm{i}} e^{\xi \sqrt{-\lambda \mathrm{i}}} \\
& +\varsigma_{11} \sqrt{\lambda(\lambda+b)} e^{-\xi \sqrt{\lambda(\lambda+b)}}-\varsigma_{12} \sqrt{\lambda(\lambda+b)} e^{\xi \sqrt{\lambda(\lambda+b)}}=0 \\
& \sigma_{21} e^{(1-\xi) \sqrt{-\lambda \mathrm{i}}}+\sigma_{22} e^{-(1-\xi) \sqrt{-\lambda \mathrm{i}}}=0 \text {, } \\
& \varsigma_{21} e^{(1-\xi) \sqrt{\lambda(\lambda+b)}}+\varsigma_{22} e^{-(1-\xi) \sqrt{\lambda(\lambda+b)}}=-\breve{\zeta}_{21} e^{(1-\xi) \sqrt{\lambda(\lambda+b)}}-\breve{\zeta}_{22} e^{-(1-\xi) \sqrt{\lambda(\lambda+b)}} \\
& =-\frac{1}{2 \sqrt{\lambda(\lambda+b)}} \sinh ((1-\xi) \sqrt{\lambda(\lambda+b)}) \\
& \sigma_{21} e^{-\xi \sqrt{-\lambda \mathrm{i}}}+\sigma_{22} e^{\xi \sqrt{-\lambda \mathrm{i}}}+\varsigma_{21} k \lambda(\lambda+b) e^{-\xi \sqrt{\lambda(\lambda+b)}} \\
& +\varsigma_{22} k \lambda(\lambda+b) e^{\xi \sqrt{\lambda(\lambda+b)}}=-k \delta_{0}(\xi) \\
& \sigma_{21} \mathrm{i} k \sqrt{-\lambda \mathrm{i}} e^{-\xi \sqrt{-\lambda \mathrm{i}}}-\sigma_{22} \mathrm{i} k \sqrt{-\lambda \mathrm{i}} e^{\xi \sqrt{-\lambda \mathrm{i}}} \\
& +\varsigma_{21}(\lambda+b) \sqrt{\lambda(\lambda+b)} e^{-\xi \sqrt{\lambda(\lambda+b)}}-\varsigma_{22}(\lambda+b) \sqrt{\lambda(\lambda+b)} e^{\xi \sqrt{\lambda(\lambda+b)}}=0 \text {. }
\end{aligned}
$$

and

$$
\begin{aligned}
& \sigma_{31} e^{(1-\xi) \sqrt{-\lambda \mathrm{i}}}+\sigma_{32} e^{-(1-\xi) \sqrt{-\lambda \mathrm{i}}}=0, \\
& \varsigma_{31} e^{(1-\xi) \sqrt{\lambda(\lambda+b)}}+\varsigma_{32} e^{-(1-\xi) \sqrt{\lambda(\lambda+b)}}=-\breve{\zeta}_{31} e^{(1-\xi) \sqrt{\lambda(\lambda+b)}}-\breve{\zeta}_{32} e^{-(1-\xi) \sqrt{\lambda(\lambda+b)}} \\
& \left.\begin{array}{l}
=-\frac{1}{2 \sqrt{\lambda(\lambda+b)}} \sinh ((1-\xi) \sqrt{\lambda(\lambda+b)}), \\
\int^{\lambda(\lambda+b)}+\varsigma_{32} \lambda k e^{\xi \sqrt{\lambda(\lambda+b)}}=0,
\end{array}\right\}, \quad \forall \xi \in[0,1] . \\
& \begin{array}{l}
\sigma_{31} e^{-\xi \sqrt{-\lambda \mathrm{i}}}+\sigma_{32} e^{\xi \sqrt{-\lambda \mathrm{i}}}+\varsigma_{31} \lambda k e^{-\xi \sqrt{\lambda(\lambda+b)}} \\
\sigma_{31} \mathrm{i} k \sqrt{-\lambda \mathrm{i}} e^{-\xi \sqrt{-\lambda \mathrm{i}}}-\sigma_{32} \mathrm{i} k \sqrt{-\lambda \mathrm{i}} e^{\xi \sqrt{-\lambda \mathrm{i}}}
\end{array} \\
& +\varsigma_{31} \sqrt{\lambda(\lambda+b)} e^{-\xi \sqrt{\lambda(\lambda+b)}}-\varsigma_{32} \sqrt{\lambda(\lambda+b)} e^{\xi \sqrt{\lambda(\lambda+b)}}=0
\end{aligned}
$$

By Cramer's rule, we can deduce from (2.4) that

$$
\begin{aligned}
& \breve{\sigma}_{11}=\frac{\operatorname{det}\left(\begin{array}{cc}
0 & 1 \\
\frac{1}{2}-\sqrt{-\lambda \mathrm{i}}
\end{array}\right)}{\operatorname{det}\left(\begin{array}{c}
1 \\
\sqrt{-\lambda \mathrm{i}}-\sqrt{-\lambda \mathrm{i}})
\end{array}\right)}=\frac{1}{4 \sqrt{-\lambda \mathrm{i}}}, \\
& \breve{\sigma}_{12}=\frac{\operatorname{det}\left(\begin{array}{cc}
1 & 0 \\
\sqrt{-\lambda \mathrm{i}} & \frac{1}{2}
\end{array}\right)}{\operatorname{det}\left(\begin{array}{c}
1 \\
\sqrt{-\lambda \mathrm{i}}-\sqrt{-\lambda \mathrm{i}})
\end{array}\right)}=-\frac{1}{4 \sqrt{-\lambda \mathrm{i}}}, \\
& \breve{\zeta}_{21}=\breve{\zeta}_{31}=\frac{\operatorname{det}\left(\begin{array}{c}
0 \\
\left.\frac{1}{2}-\sqrt{\lambda(\lambda+b)}\right)
\end{array}\right)}{\operatorname{det}\left(\begin{array}{c}
1 \\
\sqrt{\lambda(\lambda+b)}-\sqrt{\lambda(\lambda+b)})
\end{array}\right)}=\frac{1}{4 \sqrt{\lambda(\lambda+b)}}, \\
& \breve{\zeta}_{22}=\breve{\zeta}_{32}=\frac{\operatorname{det}\left(\begin{array}{c}
1 \\
\sqrt{\lambda(\lambda+b)} \frac{1}{2}
\end{array}\right)}{\operatorname{det}\left(\begin{array}{c}
1 \\
\sqrt{\lambda(\lambda+b)}-\sqrt{\lambda(\lambda+b)})
\end{array}\right)-\frac{1}{4 \sqrt{\lambda(\lambda+b)}} .} .
\end{aligned}
$$

We deduce $\sigma_{11}$ from (2.5) by Cramer's rule that

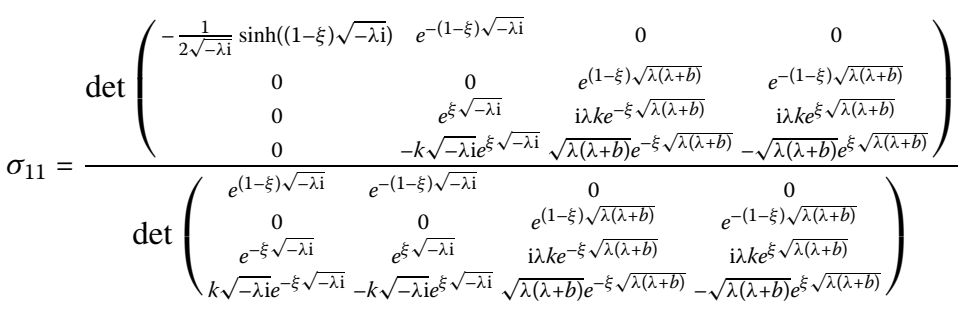




$$
\begin{aligned}
& =\frac{-\frac{1}{2 \sqrt{-\lambda \mathrm{i}}} \sinh ((1-\xi) \sqrt{-\lambda \mathrm{i})}) \operatorname{det}\left(\begin{array}{ccc}
0 & e^{(1-\xi) \sqrt{\lambda(\lambda+b)}} & e^{-(1-\xi) \sqrt{\lambda(\lambda+b)}} \\
e^{\xi \sqrt{-\lambda \mathrm{i}}} & \mathrm{i} \lambda k e^{-\xi \sqrt{\lambda(\lambda+b)}} & \mathrm{i} \lambda k e^{\xi \sqrt{\lambda(\lambda+b)}} \\
-k \sqrt{-\lambda \mathrm{i}} e^{\xi \sqrt{-\lambda \mathrm{i}}} \sqrt{\lambda(\lambda+b)} e^{-\xi \sqrt{\lambda(\lambda+b)}}-\sqrt{\lambda(\lambda+b)} e^{\xi \sqrt{\lambda(\lambda+b)}}
\end{array}\right)}{\Delta} \\
& =-\frac{e^{\xi \sqrt{-\lambda \mathrm{i}}} \sinh ((1-\xi) \sqrt{-\lambda \mathrm{i}})\left[\sqrt{\lambda(\lambda+b)} \cosh (\sqrt{\lambda(\lambda+b)})-\mathrm{i} \lambda k^{2} \sqrt{-\lambda \mathrm{i}} \sinh (\sqrt{\lambda(\lambda+b)})\right]}{\sqrt{-\lambda \mathrm{i}} \Delta} \\
& =-\frac{1}{4 \sqrt{-\lambda \mathrm{i}}}+\frac{\sqrt{\lambda(\lambda+b)} \cosh (\sqrt{\lambda(\lambda+b)})\left[e^{(2 \xi-1) \sqrt{-\lambda \mathrm{i}}}-e^{-\sqrt{-\lambda \mathrm{i}}}\right]}{2 \sqrt{-\lambda \mathrm{i}} \Delta} \\
& -\frac{\mathrm{i} \lambda k^{2} \sqrt{-\lambda \mathrm{i}} \sinh (\sqrt{\lambda(\lambda+b)})\left[e^{(2 \xi-1) \sqrt{-\lambda \mathrm{i}}}+e^{-\sqrt{-\lambda \mathrm{i}}}\right]}{2 \sqrt{-\lambda \mathrm{i}} \Delta},
\end{aligned}
$$

where $\Delta$ is given by

$$
\begin{aligned}
& \Delta=\operatorname{det}\left(\begin{array}{cccc}
e^{(1-\xi) \sqrt{-\lambda \mathrm{i}}} & e^{-(1-\xi) \sqrt{-\lambda \mathrm{i}}} & 0 & 0 \\
0 & 0 & e^{(1-\xi) \sqrt{\lambda(\lambda+b)}} & e^{-(1-\xi) \sqrt{\lambda(\lambda+b)}} \\
e^{-\xi \sqrt{-\lambda \mathrm{i}}} & e^{\xi \sqrt{-\lambda \mathrm{i}}} & \mathrm{i} \lambda k e^{-\xi \sqrt{\lambda(\lambda+b)}} & \mathrm{i} \lambda k e^{\xi \sqrt{\lambda(\lambda+b)}} \\
k \sqrt{-\lambda \mathrm{i}} e^{-\xi \sqrt{-\lambda \mathrm{i}}} & -k \sqrt{-\lambda \mathrm{i}} e^{\xi \sqrt{-\lambda \mathrm{i}}} & \sqrt{\lambda(\lambda+b)} e^{-\xi \sqrt{\lambda(\lambda+b)}} & -\sqrt{\lambda(\lambda+b)} e^{\xi \sqrt{\lambda(\lambda+b)}}
\end{array}\right) \\
& =\operatorname{det}\left(\begin{array}{cc}
e^{(1-\xi) \sqrt{-\lambda \mathrm{i}}} & e^{-(1-\xi) \sqrt{-\lambda \mathrm{i}}} \\
k \sqrt{-\lambda \mathrm{i}} e^{-\xi \sqrt{-\lambda \mathrm{i}}} & -k \sqrt{-\lambda \mathrm{i}} e^{\xi \sqrt{-\lambda \mathrm{i}}}
\end{array}\right) \operatorname{det}\left(\begin{array}{cc}
e^{(1-\xi) \sqrt{\lambda(\lambda+b)}} & e^{-(1-\xi) \sqrt{\lambda(\lambda+b)}} \\
\mathrm{i} \lambda k e^{-\xi \sqrt{\lambda(\lambda+b)}} & \mathrm{i} \lambda k e^{\xi \sqrt{\lambda(\lambda+b)}}
\end{array}\right) \\
& -\operatorname{det}\left(\begin{array}{cc}
e^{(1-\xi) \sqrt{-\lambda \mathrm{i}}} & e^{-(1-\xi) \sqrt{-\lambda \mathrm{i}}} \\
e^{-\xi \sqrt{-\lambda \mathrm{i}}} & e^{\xi \sqrt{-\lambda \mathrm{i}}}
\end{array}\right) \operatorname{det}\left(\begin{array}{cc}
e^{(1-\xi) \sqrt{\lambda(\lambda+b)}} & e^{-(1-\xi) \sqrt{\lambda(\lambda+b)}} \\
\sqrt{\lambda(\lambda+b)} e^{-\xi \sqrt{\lambda(\lambda+b)}} & -\sqrt{\lambda(\lambda+b)} e^{\xi \sqrt{\lambda(\lambda+b)}}
\end{array}\right) \\
& =\sqrt{\lambda(\lambda+b)}\left[e^{\sqrt{\lambda(\lambda+b)}+\sqrt{-\lambda \mathrm{i}}}-e^{\sqrt{\lambda(\lambda+b)}-\sqrt{-\lambda \mathrm{i}}}+e^{-\sqrt{\lambda(\lambda+b)}+\sqrt{-\lambda \mathrm{i}}}-e^{-\sqrt{\lambda(\lambda+b)}-\sqrt{-\lambda \mathrm{i}}}\right] \\
& -\mathrm{i} \lambda k^{2} \sqrt{-\lambda \mathrm{i}}\left[e^{\sqrt{\lambda(\lambda+b)}+\sqrt{-\lambda \mathrm{i}}}+e^{\sqrt{\lambda(\lambda+b)}-\sqrt{-\lambda \mathrm{i}}}-e^{-\sqrt{\lambda(\lambda+b)}+\sqrt{-\lambda \mathrm{i}}}-e^{-\sqrt{\lambda(\lambda+b)}-\sqrt{-\lambda \mathrm{i}}}\right] \\
& =4 \sqrt{\lambda(\lambda+b)} \cosh (\sqrt{\lambda(\lambda+b)}) \sinh (\sqrt{-\lambda \mathrm{i}}) \\
& -4 \mathrm{i} \lambda k^{2} \sqrt{-\lambda \mathrm{i}} \sinh (\sqrt{\lambda(\lambda+b)}) \cosh (\sqrt{-\lambda \mathrm{i}}) \text {. }
\end{aligned}
$$

Similarly, we can deduce from $(2.5)$ that $\sigma_{12}, \varsigma_{11}, \varsigma_{12}$ can be expressed as follows:

$$
\begin{aligned}
& \sigma_{12}=\frac{\operatorname{det}\left(\begin{array}{cccc}
e^{(1-\xi) \sqrt{-\lambda \mathrm{i}}} & -\frac{1}{2 \sqrt{-\lambda \mathrm{i}}} \sinh ((1-\xi) \sqrt{-\lambda \mathrm{i})} & 0 & 0 \\
0 & 0 & e^{(1-\xi) \sqrt{\lambda(\lambda+b)}} & e^{-(1-\xi) \sqrt{\lambda(\lambda+b)}} \\
e^{-\xi \sqrt{-\lambda \mathrm{i}}} & 0 & \mathrm{i} \lambda k e^{-\xi \sqrt{\lambda(\lambda+b)}} & \mathrm{i} \lambda k e^{\xi \sqrt{\lambda(\lambda+b)}} \\
k \sqrt{-\lambda \mathrm{i}} e^{-\xi \sqrt{-\lambda \mathrm{i}}} & 0 & \sqrt{\lambda(\lambda+b)} e^{-\xi \sqrt{\lambda(\lambda+b)}} & -\sqrt{\lambda(\lambda+b)} e^{\xi} \sqrt{\lambda(\lambda+b)}
\end{array}\right)}{\Delta} \\
& =\frac{e^{-\xi \sqrt{-\lambda \mathrm{i}}} \sinh ((1-\xi) \sqrt{-\lambda \mathrm{i}})\left[\sqrt{\lambda(\lambda+b)} \cosh (\sqrt{\lambda(\lambda+b)})+\mathrm{i} \lambda k^{2} \sqrt{-\lambda \mathrm{i}} \sinh (\sqrt{\lambda(\lambda+b)})\right]}{\sqrt{-\lambda \mathrm{i}} \Delta},
\end{aligned}
$$

$$
\begin{aligned}
& S_{11}= \frac{\operatorname{det}\left(\begin{array}{cccc}
e^{(1-\xi) \sqrt{-\lambda \mathrm{i}}} & e^{-(1-\xi) \sqrt{-\lambda \mathrm{i}}} & -\frac{1}{2 \sqrt{-\lambda \mathrm{i}}} \sinh ((1-\xi) \sqrt{-\lambda \mathrm{i}}) & 0 \\
0 & 0 & 0 & e^{-(1-\xi) \sqrt{\lambda(\lambda+b)}} \\
e^{-\xi \sqrt{-\lambda \mathrm{i}}} & e^{\xi \sqrt{-\lambda \mathrm{i}}} & 0 & \mathrm{i} \lambda k e^{\xi \sqrt{\lambda(\lambda+b)}} \\
k \sqrt{-\lambda \mathrm{i}} e^{-\xi \sqrt{-\lambda \mathrm{i}}}-k \sqrt{-\lambda \mathrm{i} e^{\xi} \sqrt{-\lambda \mathrm{i}}} & 0 & -\sqrt{\lambda(\lambda+b)} e^{\xi \sqrt{\lambda(\lambda+b)}}
\end{array}\right)}{\Delta}, \\
&=\frac{k e^{-(1-\xi) \sqrt{\lambda(\lambda+b)}} \sinh ((1-\xi) \sqrt{-\lambda \mathrm{i})}}{\Delta},
\end{aligned}
$$


and

$$
\begin{aligned}
& \varsigma_{12}=\frac{\operatorname{det}\left(\begin{array}{cccc}
e^{(1-\xi) \sqrt{-\lambda \mathrm{i}}} & e^{-(1-\xi) \sqrt{-\lambda \mathrm{i}}} & 0 & -\frac{1}{2 \sqrt{-\lambda \mathrm{i}}} \sinh ((1-\xi) \sqrt{-\lambda \mathrm{i})} \\
0 & 0 & e^{(1-\xi) \sqrt{\lambda(\lambda+b)}} & 0 \\
e^{-\xi \sqrt{-\lambda \mathrm{i}}} & e^{\xi \sqrt{-\lambda \mathrm{i}}} & \mathrm{i} \lambda k e^{-\xi \sqrt{\lambda(\lambda+b)}} & 0 \\
k \sqrt{-\lambda \mathrm{i}} e^{-\xi \sqrt{-\lambda \mathrm{i}}}-k \sqrt{-\lambda \mathrm{i}} e^{\xi \sqrt{-\lambda \mathrm{i}}} \sqrt{\lambda(\lambda+b)} e^{-\xi \sqrt{\lambda(\lambda+b)}} & 0
\end{array}\right)}{\Delta} \\
& =-\frac{k e^{(1-\xi) \sqrt{\lambda(\lambda+b)}} \sinh ((1-\xi) \sqrt{-\lambda \mathrm{i}})}{\Delta} .
\end{aligned}
$$

We can deduce from $(2.6)$ that $\sigma_{21}, \sigma_{22}, \varsigma_{21}, \varsigma_{22}$ can be expressed as follows:

$$
\begin{aligned}
& \sigma_{21}=\frac{k e^{-(1-\xi) \sqrt{-\lambda \mathrm{i}}}\left[2 \delta_{0}(\xi) \sqrt{\lambda(\lambda+b)} \cosh (\sqrt{\lambda(\lambda+b)})-\lambda(\lambda+b) \sinh ((1-\xi) \sqrt{\lambda(\lambda+b)})\right]}{\Delta} \\
& \sigma_{22}=-\frac{k e^{(1-\xi) \sqrt{-\lambda \mathrm{i}}}\left[2 \delta_{0}(\xi) \sqrt{\lambda(\lambda+b)} \cosh (\sqrt{\lambda(\lambda+b)})-\lambda(\lambda+b) \sinh ((1-\xi) \sqrt{\lambda(\lambda+b)})\right]}{\Delta}, \\
& \varsigma_{21}=-\frac{2 \mathrm{i} k^{2} \sqrt{-\lambda \mathrm{i}} \delta_{0}(\xi) e^{-(1-\xi) \sqrt{\lambda(\lambda+b)}} \cosh (\sqrt{-\lambda \mathrm{i}})}{(\lambda+b) \Delta} \\
& -\frac{\sinh (\sqrt{-\lambda \mathrm{i}}) \sinh ((1-\xi) \sqrt{\lambda(\lambda+b)}) e^{\xi \sqrt{\lambda(\lambda+b)}}}{\Delta} \\
& +\frac{\mathrm{i} \lambda k^{2} \sqrt{-\lambda \mathrm{i}} \cosh (\sqrt{-\lambda \mathrm{i}}) \sinh ((1-\xi) \sqrt{\lambda(\lambda+b)}) e^{\xi \sqrt{\lambda(\lambda+b)}}}{\Delta \sqrt{\lambda(\lambda+b)}}, \\
& \varsigma_{22}=\frac{2 \mathrm{i} k^{2} \sqrt{-\lambda} \mathrm{i} \delta_{0}(\xi) e^{(1-\xi) \sqrt{\lambda(\lambda+b)}} \cosh (\sqrt{-\lambda \mathrm{i}})}{(\lambda+b) \Delta} \\
& -\frac{\sinh (\sqrt{-\lambda \mathrm{i}}) \sinh ((1-\xi) \sqrt{\lambda(\lambda+b)}) e^{-\xi \sqrt{\lambda(\lambda+b)}}}{\Delta} \\
& -\frac{\mathrm{i} \lambda k^{2} \sqrt{-\lambda \mathrm{i}} \cosh (\sqrt{-\lambda \mathrm{i}}) \sinh ((1-\xi) \sqrt{\lambda(\lambda+b)}) e^{-\xi \sqrt{\lambda(\lambda+b)}}}{\Delta \sqrt{\lambda(\lambda+b)}} .
\end{aligned}
$$

We can deduce from $(2.7)$ that $\sigma_{31}, \sigma_{32}, \varsigma_{31}, \varsigma_{32}$ can be expressed as follows:

$$
\begin{aligned}
& \sigma_{31}=-\frac{\lambda k e^{-(1-\xi) \sqrt{-\lambda \mathrm{i}}} \sinh ((1-\xi) \sqrt{\lambda(\lambda+b)})}{\Delta}, \\
& \sigma_{32}=\frac{\lambda k e^{(1-\xi) \sqrt{-\lambda \mathrm{i}}} \sinh ((1-\xi) \sqrt{\lambda(\lambda+b)})}{\Delta}, \\
& \varsigma_{31}=\frac{\mathrm{i} \lambda k^{2} \sqrt{-\lambda \mathrm{i}} \cosh (\sqrt{-\lambda \mathrm{i}}) \sinh ((1-\xi) \sqrt{\lambda(\lambda+b)}) e^{\xi \sqrt{\lambda(\lambda+b)}}}{\sqrt{\lambda(\lambda+b)} \Delta} \\
& -\frac{\sinh (\sqrt{-\lambda \mathrm{i}}) \sinh ((1-\xi) \sqrt{\lambda(\lambda+b)}) e^{\xi \sqrt{\lambda(\lambda+b)}}}{\Delta}, \\
& \varsigma_{32}=-\frac{\mathrm{i} \lambda k^{2} \sqrt{-\lambda \mathrm{i}} \cosh (\sqrt{-\lambda \mathrm{i}}) \sinh ((1-\xi) \sqrt{\lambda(\lambda+b)}) e^{-\xi \sqrt{\lambda(\lambda+b)}}}{\sqrt{\lambda(\lambda+b)} \Delta} \\
& -\frac{\sinh (\sqrt{-\lambda \mathrm{i}}) \sinh ((1-\xi) \sqrt{\lambda(\lambda+b)}) e^{-\xi \sqrt{\lambda(\lambda+b)}}}{\Delta} .
\end{aligned}
$$

Let us remind that $\Delta$ in the above formulas is given explicitly by (2.13). 


\section{Proof of the main results}

We seek to obtain in this section the lower bound for $|\Delta|$ (see (2.13) for the definition of $\Delta$ ). As mentioned in Sect. 2 , we need merely consider the situation $\lambda \in i \mathbb{R}$. For the sake of clarity, we distinguish $\lambda$ into two cases.

Case $1(\lambda \in \mathbb{C} \backslash\{0\}$ and $\lambda=|\lambda| i)$ In this case, $\sqrt{\lambda(\lambda+b)}=\mathfrak{p}(|\lambda|)+\mathrm{iq}(|\lambda|)$, where

$$
\begin{array}{ll}
\mathfrak{p}(\mu)=\frac{b}{\sqrt{2\left[1+\sqrt{1+(b / \mu)^{2}}\right]}} \quad \text { and } \\
\mathfrak{q}(\mu)=\mu \sqrt{\frac{1+\sqrt{1+(b / \mu)^{2}}}{2}}, \quad \forall \mu \in(0, \infty) .
\end{array}
$$

Obviously, we have

$$
\begin{aligned}
& \mathfrak{p}(\mu)<\frac{b}{2} \quad \text { and } \quad \mathfrak{q}(\mu)>\mu, \quad \forall \mu \in(0, \infty), \quad \text { and } \\
& \mathfrak{p}(\mu)>\frac{b}{4}, \quad \forall \mu \in\left[\frac{b}{\sqrt{48}}, \infty\right) .
\end{aligned}
$$

Mainly using the triangle inequality, we can deduce from (2.13) that

$$
\begin{aligned}
|\Delta| \geq|\lambda|^{\frac{3}{2}} e^{\mathfrak{p}(|\lambda|)+\sqrt{|\lambda|}}\left\{-\left|\frac{\mathrm{i} \sqrt{\lambda(\lambda+b)}}{\lambda k^{2} \sqrt{-\lambda \mathrm{i}}}\right|\left[1+e^{-2 \sqrt{|\lambda|}}+e^{-2 \mathfrak{p}(|\lambda|)}+e^{-2 \mathfrak{p}(|\lambda|)-2 \sqrt{|\lambda|}}\right]\right. \\
\left.+\left|1-e^{-2 \sqrt{\lambda(\lambda+b)}}\right|-e^{-2 \sqrt{|\lambda|}}-e^{-2 \mathfrak{p}(|\lambda|)-2 \sqrt{|\lambda|}}\right\} .
\end{aligned}
$$

But

$$
\begin{aligned}
\mid 1-e^{-2 \sqrt{\lambda(\lambda+b)} \mid} & \geq 1-e^{-2 \mathfrak{p}(|\lambda|)} \\
& \geq 1-\exp \left(-\frac{b}{2}\right) \\
& >\frac{b}{2+b} \quad \text { whenever }|\lambda| \geq \frac{b}{\sqrt{48}},
\end{aligned}
$$

where the " $\geq$ " in the second line follows if and only if $|\lambda| \geq \frac{b}{\sqrt{48}}$, and $\mathfrak{p}(\cdot)$ is given by (3.1). And similarly, we have

$$
\begin{aligned}
& \left|\frac{\mathrm{i} \sqrt{\lambda(\lambda+b)}}{\lambda k^{2} \sqrt{-\lambda \mathrm{i}}}\right|\left[1+e^{-2 \sqrt{|\lambda|}}+e^{-2 \mathfrak{p}(|\lambda|)}+e^{-2 \mathfrak{p}(|\lambda|)-2 \sqrt{|\lambda|}]}\right. \\
& \quad \leq \frac{4}{k^{2}} \sqrt{\frac{1}{|\lambda|}+\frac{b}{|\lambda|^{2}}} \leq \frac{b}{3(2+b)} \quad \text { whenever }|\lambda| \geq \max \left(b, \frac{288(b+2)^{2}}{k^{4} b^{2}}\right)
\end{aligned}
$$

and

$$
e^{-2 \sqrt{|\lambda|}}+e^{-2 \mathfrak{p}(|\lambda|)-2 \sqrt{|\lambda|}} \leq \frac{b}{3(2+b)} \quad \text { whenever }|\lambda| \geq \frac{6(2+b)}{b} .
$$


Therefore,

$$
\begin{aligned}
& |\Delta| \geq \frac{b}{3(2+b)}|\lambda|^{\frac{3}{2}} e^{\sqrt{|\lambda|}}, \\
& \forall \lambda \in \mathbb{C} \text { with } \lambda=|\lambda| \text { i and with }|\lambda| \geq \max \left(b, \frac{288(b+2)^{2}}{k^{4} b^{2}}, \frac{6(2+b)}{b}\right) .
\end{aligned}
$$

Case $2(\lambda \in \mathbb{C} \backslash\{0\}$ and $\lambda=-|\lambda| \mathrm{i})$ In this case, $\sqrt{\lambda(\lambda+b)}=\mathfrak{p}(|\lambda|)-\mathrm{i} \mathfrak{q}(|\lambda|)$, where $\mathfrak{p}$ and $\mathfrak{q}$ are given by (3.1). Substitute this into (2.13) to obtain

$$
\begin{aligned}
\Delta= & 4 \mathrm{i}[\mathfrak{p}(|\lambda|)-\mathrm{iq}(|\lambda|)] \cosh (\mathfrak{p}(|\lambda|)-\mathrm{i} \mathfrak{q}(|\lambda|)) \sin (\sqrt{|\lambda|}) \\
& -4 \mathrm{i} k^{2}|\lambda|^{\frac{3}{2}} \sinh (\mathfrak{p}(|\lambda|)-\mathrm{i} \mathfrak{q}(|\lambda|)) \cos (\sqrt{|\lambda|}) \\
= & 4 \mathrm{i}[\mathfrak{p}(|\lambda|)-\mathrm{iq}(|\lambda|)] \\
& \times[\cosh (\mathfrak{p}(|\lambda|)) \cos (\mathfrak{q}(|\lambda|))-\mathrm{i} \sinh (\mathfrak{p}(|\lambda|)) \sin (\mathfrak{q}(|\lambda|))] \sin (\sqrt{|\lambda|}) \\
& -4 \mathrm{i} k^{2}|\lambda|^{\frac{3}{2}}[\sinh (\mathfrak{p}(|\lambda|)) \cos (\mathfrak{q}(|\lambda|))-\mathrm{i} \cosh (\mathfrak{p}(|\lambda|)) \sin (\mathfrak{q}(|\lambda|))] \cos (\sqrt{|\lambda|}) \\
= & 4 \alpha(|\lambda|)+4 \beta(|\lambda|) \mathrm{i} .
\end{aligned}
$$

Here $\alpha$ and $\beta$ are given explicitly as

$$
\left.\begin{array}{rl}
\alpha(\mu)= & \sin (\sqrt{\mu})[\mathfrak{p}(\mu) \sinh (\mathfrak{p}(\mu)) \sin (\mathfrak{q}(\mu))+\mathfrak{q}(\mu) \cosh (\mathfrak{p}(\mu)) \cos (\mathfrak{q}(\mu))] \\
& -k^{2} \mu^{\frac{3}{2}} \cos (\sqrt{\mu}) \cosh (\mathfrak{p}(\mu)) \sin (\mathfrak{q}(\mu)), \\
\beta(\mu)= & \sin (\sqrt{\mu})[\mathfrak{p}(\mu) \cosh (\mathfrak{p}(\mu)) \cos (\mathfrak{q}(\mu))-\mathfrak{q}(\mu) \sinh (\mathfrak{p}(\mu)) \sin (\mathfrak{q}(\mu))] \\
& -k^{2} \mu^{\frac{3}{2}} \cos (\sqrt{\mu}) \sinh (\mathfrak{p}(\mu)) \cos (\mathfrak{q}(\mu))
\end{array}\right\}, \quad \forall \mu \in[0, \infty),
$$

and satisfy

$$
\begin{aligned}
|\alpha(\mu)|^{2}+|\beta(\mu)|^{2}= & \left.\left.\left|\mathfrak{q}(\mu) \sin (\sqrt{\mu}) \cos (\mathfrak{q}(\mu))-k^{2}\right| \lambda\right|^{\frac{3}{2}} \cos (\sqrt{\mu}) \sin (\mathfrak{q}(\mu))\right|^{2} \\
& -\frac{\mathfrak{p}(\mu) k^{2} \mu^{\frac{3}{2}} \sin (2 \sqrt{\mu}) \sinh (2 \mathfrak{p}(\mu))}{2} \\
& +\frac{[\cosh (2 \mathfrak{p}(\mu))-1]\left[|\mathfrak{q}(\mu)|^{2} \sin ^{2}(\sqrt{\mu})+k^{4} \mu^{3} \cos ^{2}(\sqrt{\mu})\right]}{2} \\
& +\frac{|\mathfrak{p}(\mu)|^{2} \sin ^{2}(\sqrt{\mu})[\cosh (2 \mathfrak{p}(\mu))+\cos (2 \mathfrak{q}(\mu))]}{2} \\
\geq & \frac{b^{2}\left[\mu^{2} \sin ^{2}(\sqrt{\mu})+\mu^{2} \cos ^{2}(\sqrt{\mu})\right]}{16}-\frac{b e^{b} k^{2} \mu^{\frac{3}{2}}}{8} \\
= & \frac{\mu^{2} b^{2}}{32}+\frac{\mu^{\frac{3}{2}} b^{2}}{32}\left(\sqrt{\mu}-\frac{4 e^{b} k^{2}}{b}\right) \\
\geq & \frac{\mu^{2} b^{2}}{32} \quad \text { whenever } \mu \geq \max \left(\frac{16 e^{2 b} k^{4}}{b^{2}}, \frac{1}{k^{4}}, \frac{b}{\sqrt{48}}\right)
\end{aligned}
$$


where the "=" in the first line follows from a series of elementary calculations and rearrangements, the " $\geq$ " in the third line follows from (3.2) and

$$
\begin{aligned}
& \mu^{3} \geq \frac{\mu^{2}}{k^{4}} \quad \text { whenever } \mu \geq \frac{1}{k^{4}}, \quad \text { and } \\
& \left|\mathfrak{p}(\mu) k^{2} \mu^{\frac{3}{2}} \sin (2 \sqrt{\mu}) \sinh (2 \mathfrak{p}(\mu))\right| \leq \frac{b e^{b} k^{2} \mu^{\frac{3}{2}}}{4}, \quad \forall \mu \in(0, \infty) .
\end{aligned}
$$

By (3.10), we deduce from (3.8) that

$$
\begin{aligned}
|\Delta|^{2} & =16|\alpha(|\lambda|)|^{2}+16|\beta(|\lambda|)|^{2} \\
& \geq \frac{b^{2}|\lambda|^{2}}{2} \quad \text { with } \lambda=-|\lambda| i,|\lambda| \geq \max \left(\frac{16 e^{2 b} k^{4}}{b^{2}}, \frac{1}{k^{4}}, \frac{b}{\sqrt{48}}\right) .
\end{aligned}
$$

Having the above analysis results at our disposal, we are now in a position to prove the main results.

Proof of Theorem 1.2 It is equivalent to proving

$$
\left.\begin{array}{rl}
\|f\|_{L^{2}(0,1)}^{2} & \leq C\langle\lambda\rangle^{4}\left(\|\phi\|_{L^{2}(0,1)}^{2}+\|\psi\|_{H^{1}(0,1)}^{2}+\|\eta\|_{L^{2}(0,1)}^{2}\right), \\
\|g\|_{H^{1}(0,1)}^{2} & \leq C\langle\lambda\rangle^{4}\left(\|\phi\|_{L^{2}(0,1)}^{2}+\|\psi\|_{H^{1}(0,1)}^{2}+\|\eta\|_{L^{2}(0,1)}^{2}\right), \\
\|h\|_{L^{2}(0,1)}^{2} & \leq C\langle\lambda\rangle^{4}\left(\|\phi\|_{L^{2}(0,1)}^{2}+\|\psi\|_{H^{1}(0,1)}^{2}+\|\eta\|_{L^{2}(0,1)}^{2}\right)
\end{array}\right\}, \quad \forall \lambda \in \mathrm{i} \mathbb{R}
$$

where $(f, g, h)$ and $(\phi, \psi, \eta)$ are related by $(2.2)$.

Let us consider first the term $\int_{0}^{1} G^{2}(x, \xi) \psi(\xi) d \xi$. Combine (2.2), (2.3), (2.19), and (2.20), to obtain

$$
\begin{aligned}
\widehat{\psi}(x)= & \int_{0}^{1} G^{2}(x, \xi) \psi(\xi) d \xi \\
= & -\frac{4 \mathrm{i} k^{2} \sqrt{-\lambda \mathrm{i}} \psi(0) \sinh ((x-1) \sqrt{\lambda(\lambda+b)}) \cosh (\sqrt{-\lambda \mathrm{i}})}{(\lambda+b) \Delta} \\
& -\frac{2 \sinh (\sqrt{-\lambda \mathrm{i}}) \cosh (x \sqrt{\lambda(\lambda+b)})}{\Delta} \int_{0}^{1} \psi(\xi) \sinh ((1-\xi) \sqrt{\lambda(\lambda+b)}) d \xi \\
& +\frac{2 \mathrm{i} \lambda k^{2} \sqrt{-\lambda \mathrm{i}} \cosh (\sqrt{-\lambda \mathrm{i}}) \sinh (x \sqrt{\lambda(\lambda+b)})}{\Delta \sqrt{\lambda(\lambda+b)}} \\
& \times \int_{0}^{1} \psi(\xi) \sinh ((1-\xi) \sqrt{\lambda(\lambda+b)}) d \xi \\
& +\frac{1}{2 \sqrt{\lambda(\lambda+b)}} \int_{0}^{x} \psi(\xi) \sinh ((x-\xi) \sqrt{\lambda(\lambda+b)}) d \xi .
\end{aligned}
$$

The derivative $\widehat{\psi}^{\prime}$ of $\widehat{\psi}$ reads

$$
\begin{aligned}
\widehat{\psi}^{\prime}(x)= & -\frac{4 \mathrm{i} k^{2} \sqrt{-\lambda \mathrm{i}} \psi(0) \sqrt{\lambda(\lambda+b)} \cosh ((x-1) \sqrt{\lambda(\lambda+b)}) \cosh (\sqrt{-\lambda \mathrm{i}})}{(\lambda+b) \Delta} \\
& -\frac{2 \sinh (\sqrt{-\lambda \mathrm{i}}) \sqrt{\lambda(\lambda+b)} \sinh (x \sqrt{\lambda(\lambda+b)})}{\Delta}
\end{aligned}
$$




$$
\begin{aligned}
& \times \int_{0}^{1} \psi(\xi) \sinh ((1-\xi) \sqrt{\lambda(\lambda+b)}) d \xi \\
& +\frac{2 \mathrm{i} \lambda k^{2} \sqrt{-\lambda \mathrm{i}} \cosh (\sqrt{-\lambda \mathrm{i}}) \cosh (x \sqrt{\lambda(\lambda+b)})}{\Delta} \\
& \times \int_{0}^{1} \psi(\xi) \sinh ((1-\xi) \sqrt{\lambda(\lambda+b)}) d \xi \\
& +\frac{1}{2} \int_{0}^{x} \psi(\xi) \cosh ((x-\xi) \sqrt{\lambda(\lambda+b)}) d \xi
\end{aligned}
$$

Since $g \in H^{1}(0,1)$ satisfies $g(1)=0$ in the trace sense, it suffices to estimate $\left\|g^{\prime}\right\|_{L^{2}(0,1)}$ instead of $\|g\|_{H^{1}(0,1)}$. Therefore, we only need to analyze $\left\|\widehat{\psi}^{\prime}\right\|_{L^{2}(0,1)}$.

By a density argument, we can prove

$$
|\psi(0)| \leq\|\psi\|_{H^{1}(0,1)}
$$

By Young's inequality (see [14, Theorem 2.24, p. 33]), we have

$$
\begin{aligned}
& \int_{0}^{1}\left|\int_{0}^{x} \psi(\xi) \cosh ((x-\xi) \sqrt{\lambda(\lambda+b)}) d \xi\right|^{2} d x \\
& \quad \leq\|\psi\|_{L^{2}(0,1)}^{2}\left[\int_{0}^{1}|\cosh (x \sqrt{\lambda(\lambda+b)})| d x\right]^{2} \leq e^{b}\|\psi\|_{L^{2}(0,1)}^{2}
\end{aligned}
$$

where the " $\leq$ " in the second line follows from

$$
\begin{aligned}
{\left[\int_{0}^{1}|\cosh (x \sqrt{\lambda(\lambda+b)})| d x\right]^{2} } & \leq \max _{x \in[0,1]}|\cosh (x \sqrt{\lambda(\lambda+b)})|^{2} \\
& \leq \frac{\cosh (2 \mathfrak{p}(|\lambda|))+\cos (2 \mathfrak{q}(|\lambda|))}{2}<e^{b}
\end{aligned}
$$

in which we used (3.2) when we establish the last " $<$ ".

Mainly using Hölder's inequality, we have

$$
\left\{\begin{array}{l}
\int_{0}^{1}|\cosh ((x-1) \sqrt{\lambda(\lambda+b)})|^{2} d x \\
\quad=\frac{1}{2} \int_{0}^{1}[\cosh (2(x-1) \mathfrak{p}(|\lambda|))+\cos (2(x-1) \mathfrak{q}(|\lambda|))] d x \\
\quad \leq \max _{x \in[0,1]} \frac{\cosh (2(x-1) \mathfrak{p}(|\lambda|))+\cos (2(x-1) \mathfrak{q}(|\lambda|))}{2}<e^{b} \\
\int_{0}^{1}|\sinh (x \sqrt{\lambda(\lambda+b)})|^{2} d x<e^{b} \quad \text { and } \quad \int_{0}^{1}|\cosh (x \sqrt{\lambda(\lambda+b)})|^{2} d x<e^{b} \\
\left|\int_{0}^{1} \psi(\xi) \sinh ((1-\xi) \sqrt{\lambda(\lambda+b)}) d \xi\right|^{2} \\
\quad \leq\|\psi\|_{L^{2}(0,1)}^{2} \max _{\xi \in[0,1]}|\sinh ((1-\xi) \sqrt{\lambda(\lambda+b)})|^{2} \leq e^{b}\|\psi\|_{L^{2}(0,1)}^{2}
\end{array}\right.
$$

By some routine calculations, we have

$$
\begin{aligned}
& \left.\begin{array}{l}
\left|\frac{\sqrt{-\lambda \mathrm{i}} \sqrt{\lambda(\lambda+b)} \cosh (\sqrt{-\lambda \mathrm{i}})}{(\lambda+b) \Delta}\right| \leq \frac{6(2+b)}{b \sqrt{|\lambda|}}, \\
\left|\frac{\sinh (\sqrt{-\lambda \mathrm{i}}) \sqrt{\lambda(\lambda+b)}}{\Delta}\right| \leq \frac{6(2+b)}{b}, \\
\left|\frac{\lambda \sqrt{-\lambda \mathrm{i}} \cosh (\sqrt{-\lambda \mathrm{i}})}{\Delta}\right| \leq \frac{3(2+b) \sqrt{|\lambda|}}{b}
\end{array}\right\} \\
& \text { whenever }|\lambda| \geq \max \left(\frac{16 e^{2 b} k^{4}}{b^{2}}, \frac{1}{k^{4}}, b, \frac{288(b+2)^{2}}{k^{4} b^{2}}, \frac{6(2+b)}{b}\right) \text {. }
\end{aligned}
$$


(3.14), together with (3.15), (3.16), (3.17), and (3.18), implies

$$
\left\|(\lambda+b) \widehat{\psi}^{\prime}\right\|_{L^{2}(0,1)}^{2} \leq \frac{9216\left(1+k^{4}\right)(2+b)^{2} e^{3 b}}{b^{2}}|\lambda|^{3}\|\psi\|_{H^{1}(0,1)}^{2}
$$

whenever $\lambda \in \mathbb{i} \mathbb{R}$ satisfies $|\lambda| \geq \max \left(\frac{16 e^{2 b} k^{4}}{b^{2}}, \frac{1}{k^{4}}, b, \frac{288(b+2)^{2}}{k^{4} b^{2}}, \frac{6(2+b)}{b}\right)$.

Applying the approach used in deducing (3.19) from (3.14) via the "steps" (3.15), (3.16), (3.17), and (3.18), we can prove

$$
\|g\|_{H^{1}(0,1)}^{2} \leq C\langle\lambda\rangle^{3}\left(\|\phi\|_{L^{2}(0,1)}^{2}+\|\psi\|_{H^{1}(0,1)}^{2}+\|\eta\|_{L^{2}(0,1)}^{2}\right)
$$

which, together with $(2.2)_{3}$, implies

$$
\|h\|_{L^{2}(0,1)}^{2} \leq C\langle\lambda\rangle^{4}\left(\|\phi\|_{L^{2}(0,1)}^{2}+\|\psi\|_{H^{1}(0,1)}^{2}+\|\eta\|_{L^{2}(0,1)}^{2}\right) .
$$

We can also prove

$$
\begin{aligned}
& \int_{0}^{1}\left|\int_{0}^{1} F^{2}(x, \xi) \psi(\xi) d \xi+\int_{0}^{1} F^{3}(x, \xi) \eta(\xi) d \xi\right|^{2} d x \\
& \quad \leq C\langle\lambda\rangle^{2}\left(\|\psi\|_{H^{1}(0,1)}^{2}+\|\eta\|_{L^{2}(0,1)}^{2}\right)
\end{aligned}
$$

where the constant $C>0$ is independent of $(\phi, \psi, \eta)$ and $\lambda$.

Now it remains to analyze the term $\int_{0}^{1} F^{1}(x, \xi) \phi(\xi) d \xi$. But

$$
\begin{aligned}
\int_{0}^{1} F^{1}(x, \xi) \phi(\xi) d \xi= & -\frac{\sqrt{\lambda(\lambda+b)} \cosh (\sqrt{\lambda(\lambda+b)})}{\sqrt{-\lambda \mathrm{i}}} \\
& \times \int_{x}^{1} \frac{\phi(\xi)[\cosh ((1+x-\xi) \sqrt{-\lambda \mathrm{i}})-\cosh ((1-x-\xi) \sqrt{-\lambda \mathrm{i}})]}{\Delta} d \xi \\
& +\frac{\mathrm{i} \lambda k^{2} \sqrt{-\lambda \mathrm{i}} \sinh (\sqrt{\lambda(\lambda+b)})}{\sqrt{-\lambda \mathrm{i}}} \\
& \times \int_{x}^{1} \frac{\phi(\xi)[\sinh ((1+x-\xi) \sqrt{-\lambda \mathrm{i}})-\sinh ((1-x-\xi) \sqrt{-\lambda \mathrm{i}})]}{\Delta} d \xi \\
& +\frac{e^{-x \sqrt{-\lambda \mathrm{i}}}\left[\sqrt{\lambda(\lambda+b)} \cosh (\sqrt{\lambda(\lambda+b)})+\mathrm{i} \lambda k^{2} \sqrt{-\lambda \mathrm{i}} \sinh (\sqrt{\lambda(\lambda+b)})\right]}{\sqrt{-\lambda \mathrm{i}}} \\
& \times \int_{0}^{x} \frac{\phi(\xi) \sinh ((1-\xi) \sqrt{-\lambda \mathrm{i}})}{\Delta} d \xi \\
& -\frac{1}{4 \sqrt{-\lambda \mathrm{i}} \int_{0}^{x} \phi(\xi) e^{-(x-\xi) \sqrt{-\lambda \mathrm{i}}} d \xi} \\
& +\frac{\sqrt{\lambda(\lambda+b)} \cosh (\sqrt{\lambda(\lambda+b)}) e^{(x-1) \sqrt{-\lambda \mathrm{i}}}}{\sqrt{-\lambda \mathrm{i}}} \\
& \times \int_{0}^{x} \frac{\phi(\xi) \sinh (\xi \sqrt{-\lambda \mathrm{i})} d \xi .}{\Delta}
\end{aligned}
$$


To provide in detail a way to analyze $\int_{0}^{1} F^{1}(x, \xi) \phi(\xi) d \xi$, we continue as follows:

$$
\begin{aligned}
& \left|\int_{x}^{1} \frac{\phi(\xi)[\cosh ((1+x-\xi) \sqrt{-\lambda \mathrm{i}})-\cosh ((1-x-\xi) \sqrt{-\lambda \mathrm{i}})]}{\Delta} d \xi\right|^{2} \\
& \quad \leq 2 \int_{x}^{1}|\phi(\xi)|^{2} d \xi \int_{x}^{1}\left[\left|\frac{\cosh ((1+x-\xi) \sqrt{-\lambda \mathrm{i}})}{\Delta}\right|^{2}+\left|\frac{\cosh ((1-x-\xi) \sqrt{-\lambda \mathrm{i})}}{\Delta}\right|^{2}\right] d \xi \\
& \quad \leq \frac{C}{\langle\lambda\rangle^{2}} \int_{x}^{1}|\phi(\xi)|^{2} d \xi .
\end{aligned}
$$

Employing the same idea, we analyze the rest of (3.23) term-by-term, and then collect all the information together to obtain

$$
\int_{0}^{1}\left|\int_{0}^{1} F^{1}(x, \xi) \phi(\xi) d \xi\right|^{2} d x \leq C\|\phi\|_{L^{2}(0,1)}^{2}
$$

This, together with (3.22), implies

$$
\|f\|_{L^{2}(0,1)}^{2} \leq C\langle\lambda\rangle^{2}\left(\|\phi\|_{L^{2}(0,1)}^{2}+\|\psi\|_{H^{1}(0,1)}^{2}+\|\eta\|_{L^{2}(0,1)}^{2}\right),
$$

where the constant $C>0$ is independent of $(\phi, \psi, \eta)$ and $\lambda$.

Combining (3.20), (3.21), and (3.24), we know that (3.12) is proved, so is Theorem 1.2.

\section{Concluding comments and an open question}

By analyzing carefully Green's functions for boundary value problems associated with ordinary differential equations (i.e., (2.1)), we prove that the infinitesimal generator of the semigroup associated with system (1.1) satisfies the resolvent estimate (1.9), thereby proving that the energy of system (1.1) decays polynomially.

Having a very simple underlying idea, our method is based on Green's functions and relies on heavy calculations. Our method can be modified to treat other transmission systems of 1-D partial differential equations where one of the equations is damped in the whole interval. However, according to the deductions based on our idea, it seems very hard to find the optimal decay rate of the energy of system (1.1). Therefore, one of our next concerns is to understand better the following question.

Open question Could the decay rate $(t+1)^{-1}$ given in estimate (1.8) be improved?

As indicated before, the above question seems difficult to solve with merely the method used in this paper. To close this section, we prove by a contradiction argument that the energy $E$ (defined in (1.6)) can NOT decay exponentially. Assume to the contrary that $E(t)$ decays exponentially, or equivalently, there exists a pair $\left(M_{0}, \gamma_{0}\right) \in(0, \infty)^{2}$ such that, for every $w \in H$,

$$
\left\|e^{t A} w\right\|_{H} \leq M_{0} e^{-\gamma_{0} t}\|w\|_{H}, \quad \forall t \in[0, \infty)
$$

where $H$ is given by (1.2), and $A$ by (1.3).

Write, for every $\lambda_{0} \in \mathbb{C}$ with $\gamma<\Re \mathfrak{e} \lambda<0$,

$$
R_{\lambda} w=\int_{0}^{\infty} e^{-\lambda t} e^{t A} w d t, \quad \forall w \in H .
$$


By (4.1), $R_{\lambda}$ is well defined and belongs to $\mathcal{L}(H)$. Moreover, by (4.2), $R_{\lambda}$ satisfies

$$
\left(\lambda \operatorname{id}_{H}-A\right) R_{\lambda} w=w, \quad \forall w \in H, \quad \text { and } \quad R_{\lambda}\left(\lambda \operatorname{id}_{H}-A\right) w=w, \quad \forall w \in \mathcal{D}(A) .
$$

Therefore, $\lambda$ belongs to $\rho(A)$, the resolvent set of $A$, and moreover, $R_{\lambda}=R(\lambda ; A)$, the resolvent of $A$.

Thus, we proved just now that $\lambda$ belongs to $\rho(A)$ whenever $\lambda \in \mathbb{C}$ satisfies $\gamma_{0}<\Re \mathfrak{e} \lambda<0$. This contradicts $(1.7)_{2}$. The proof is complete.

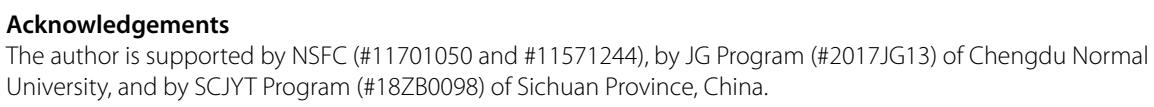

List of abbreviations

Not applicable.

Availability of data and materials

Not applicable.

Ethics approval and consent to participate

Not applicable.

Competing interests

There is no conflict of interest in this paper.

\section{Consent for publication}

Not applicable.

\section{Authors' contributions}

This piece of work is credited to CW. Author read and approved the final manuscript.

\section{Endnote}

a Throughout this paper, $\langle\cdot\rangle=\sqrt{1+|\cdot|^{2}}$

\section{Publisher's Note}

Springer Nature remains neutral with regard to jurisdictional claims in published maps and institutional affiliations.

Received: 9 August 2017 Accepted: 16 April 2018 Published online: 24 April 2018

\section{References}

1. Lu, L., Wang, J.-M.: Transmission problem of Schrödinger and wave equation with viscous damping. Appl. Math. Lett. $54,7-14(2016)$

2. Zuazua, E.: Remarks on the controllability of the Schrödinger equation. In: Quantum Control: Mathematical and Numerical Challenges. CRM Proc. Lecture Notes, vol. 33, pp. 193-211. Am. Math. Soc., Providence (2003)

3. Machtyngier, E.: Exact controllability for the Schrödinger equation. SIAM J. Control Optim. 32, 24-34 (1994)

4. Machtyngier, E., Zuazua, E.: Stabilization of the Schrödinger equation. Port. Math. 51, 243-256 (1994)

5. Phung, K.-D.: Observability and control of Schrödinger equation. SIAM J. Control Optim. 40, 211-230 (2001)

6. Lebeau, G.: Contrôle de l'équation de Schrödinger. J. Math. Pures Appl. 71, 267-291 (1992)

7. Guo, B.Z., Shao, Z.C.: Regularity of a Schrödinger equation with Dirichlet control and collocated observation. Syst. Control Lett. 54, 1135-1142 (2005)

8. Dautray, R., Lions, J.L.: Analyse Mathématique et Calcul Numérique pour les Sciences et les Techniques, vol. 1. Masson, Paris (1984)

9. Lagnese, J.: Boundary Stabilization of Thin Plates. SIAM Studies in Appl. Math., vol. 10. SIAM, Philadelphia (1989)

10. Krstic, M., Guo, B.Z., Smyshlyaev, A.: Boundary controllers and observers for the linearized Schrödinger equation. SIAM J. Control Optim. 49, 1479-1497 (2011)

11. Liu, Z., Rao, B.: Characterization of polynomial decay rate for the solution of linear evolution equation. Z. Angew. Math. Phys. 56, 630-644 (2005)

12. Burq, N.: Décroissance de l'énergie locale de l'équation des ondes pour le problème extérieur et absence de résonance au voisinage du réel. Acta Math. 180(1), 1-29 (1998)

13. Borichev, A., Tomilov, Y.: Optimal polynomial decay of functions and operator semigroups. Math. Ann. 347(2), 455-478 (2010)

14. Adams, R.A., Fournier, J.J.F.: Sobolev Spaces, 2nd edn. Pure and Applied Mathematics (Amsterdam), vol. 140 Elsevier/Academic Press, Amsterdam (2003) 